\title{
A summary of surveillance, morbidity and microbiology of laboratory-confirmed cases of infant botulism in Canada, 1979-2019
}

\author{
Richard Harris ${ }^{1}$, Christine Tchao², Natalie Prystajecky ${ }^{2,3}$, Jennifer Cutler ${ }^{4}$, John W Austin ${ }^{1 \star}$
}

\begin{abstract}
Background: Infant botulism is a rare toxicoinfectious disease caused by colonization of the infant's intestine with botulinum neurotoxin-producing clostridia (i.e. Clostridium botulinum or neurotoxigenic strains of $C$. butyricum or $C$. baratii). Our goal was to examine data from laboratory-confirmed cases of infant botulism reported in Canada to summarize incidence over time, over geographic distribution by province or territory, and by sex, and to compare these parameters with data from the Canadian Notifiable Disease Surveillance System (CNDSS). The average age of onset, serotype of botulinum neurotoxin (BoNT), case outcomes, length of hospitalization and suitability of clinical specimens for laboratory confirmation were also determined.
\end{abstract}

Methods: We examined laboratory records from the Health Canada Botulism Reference Service and the British Columbia Centre for Disease Control (BCCDC) Public Health Laboratory. The Discharge Abstract Database (DAD) and the Hospital Morbidity Database (HMDB) of the Canadian Institute of Health Information (CIHI) were queried for data on hospitalization of infant botulism cases. The CNDSS was queried for data on reported cases of infant botulism.

Results: From 1979 to 2019, 63 laboratory-confirmed cases of infant botulism were confirmed by the Health Canada Botulism Reference Service and the BCCDC Public Health Laboratory for an annual rate of 4.30 cases per million live births. From 1983 to 2018, 57 cases of infant botulism were reported to the CNDSS. Of the 63 cases confirmed by the reference laboratories, the median age of onset was 16 weeks with a range of 2 to 52 weeks. The majority of cases were type $A(76 \%)$ and $B(21 \%)$, with single cases of type $F$ and type $A B$. Of the 23 laboratoryconfirmed cases with matched hospital records, 13 were transferred to special care and eight needed ventilator support; no deaths were reported.

Conclusion: Spores of $C$. botulinum are present naturally in the environment, thus diagnosis of infant botulism does not require a history of exposure to high-risk foods such as honey. Stool samples are the most useful diagnostic specimen.
This work is licensed under a Creative Commons Attribution 4.0 International License.

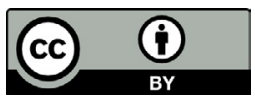

Affiliations

${ }^{1}$ Botulism Reference Service for Canada, Health Canada, Ottawa, ON

${ }^{2}$ British Columbia Centre for Disease Control Public Health Laboratory, Vancouver, BC

${ }^{3}$ Department of Pathology and Laboratory Medicine, University of British Columbia, Vancouver, BC

${ }^{4}$ Public Health Agency of Canada, Guelph, ON

\section{*Correspondence:}

john.austin@canada.ca

Suggested citation: Harris R, Tchao C, Prystajecky N, Cutler J, Austin JW. A summary of surveillance, morbidity and microbiology of laboratory-confirmed cases of infant botulism in Canada, 1979-2019. Can Commun Dis Rep 2021;47(7/8):322-8. https://doi.org/10.14745/ccdr.v47i78a05

Keywords: Clostridium botulinum, Clostridium butyricum, Clostridium baratii, incidence rate, geographic distribution, Canada, serotype, environmental source, botulinum neurotoxin

\section{Introduction}

Botulism is a neuroparalytic disease caused by exposure to botulinum neurotoxin (BoNT). Manifestations of botulism are classified according to the route of exposure to BoNTs. Foodborne botulism is an intoxication resulting from the ingestion of BoNT in food or beverages that supported the growth of Clostridium botulinum (1). Wound botulism and intestinal toxemia botulism occur when $C$. botulinum spores colonize an infected wound or the adult intestinal tract, respectively, and release BoNTs in situ $(2,3)$. 
Infant botulism, first described in $1976(4,5)$, is a form of intestinal toxemia botulism that occurs in children younger than one year. Infants are particularly susceptible to $C$. botulinum intestinal colonization because of the immaturity of their gut microbiota, whereas children over one year old can ingest C. botulinum spores without colonization $(6,7)$. In rare instances, infant botulism has been caused by BoNT-producing species C. butyricum type E (8-10) and C. baratii type F (11-14).

The clinical spectrum of infant botulism includes a wide range of severities from subclinical to fulminant. Symptoms may include, but are not limited to, hypotonia, weak suck, dysphagia, constipation, weak cry and diaphragm weakness that may require that the infant receives mechanical ventilation. Almost all cases of infant botulism are caused by Group I C. botulinum that produce BoNT type A or type B. Type A generally has a more severe clinical presentation $(6,7)$.

Infant botulism is treated with Botulism Immune Globulin Intravenous (BIG-IV or BabyBIG) antitoxin that binds to and neutralizes circulating BoNT in the bloodstream (15). It is generally recommended that infants are treated with BabyBIG as soon as possible based on a physician's differential diagnosis. Laboratory confirmation of infant botulism is based on detection of viable $C$. botulinum in stool or detection of BoNT in stool or serum.

There have been few published reports of infant botulism cases in Canada (16-20). Here we present a summary of infant botulism in Canada from 1979 to 2019, including incidence over reporting period, geographic distribution by province and territory, patient age and sex, serotype and group of $C$. botulinum involved, food or environmental source identification (where possible), as well as preferred clinical specimens for detection of BoNT and viable C. botulinum. Hospital records that match laboratory-confirmed cases demonstrate clinical outcomes of the disease.

\section{Methods}

\section{Microbiology laboratory and national surveillance data}

We examined two independent laboratory databases for laboratory-confirmed cases of infant botulism from 1979 to 2019. These databases are maintained by the Botulism Reference Service (BRS) for Canada at Health Canada, Ottawa, Ontario, and the British Columbia Centre for Disease Control (BCCDC) Public Health Laboratory, in Vancouver, British Columbia. The Health Canada BRS receives and tests clinical and food specimens associated with suspect cases of botulism from all provinces and territories when requested. The BCCDC laboratory maintains its capacity to test specimens from British Columbia, and has also tested specimens from the Yukon. Thus, these two databases do not overlap and, when combined, represent all the laboratory-confirmed cases of botulism in Canada.
We extracted information on patient age and sex, date and location of diagnosis, implicated source of $C$. botulinum, group of $C$. botulinum and BoNT serotype. The rates of disease per million live births were calculated using data from the United Nations Statistics Division and Statistics Canada $(21,22)$.

We used the national case definition for confirmed cases of infant botulism to ensure consistency in data recording: "laboratory confirmation with symptoms compatible with botulism in a person less than one year of age [with] detection of botulinum toxin in stool or serum or isolation of $C$. botulinum from the patient's stool or at autopsy" (23). Cases meeting this definition were extracted from the Canadian Notifiable Disease Surveillance System (CNDSS) and included the reporting year, province/territory, age group and sex. The CNDSS maintains basic surveillance on nationally notifiable diseases by collecting voluntarily submitted data from provinces and territories. CNDSS data was compared to laboratory data for completeness.

\section{Laboratory confirmation of clinical cases}

Detection of BoNT and isolation of viable $C$. botulinum from environmental and clinical specimens were performed according to Health Canada method MFHPB-16 (24). BoNT serotype was determined by neutralizing toxicity with serotype-specific antibodies provided by the United States (US) Centers for Disease Control. The group of C. botulinum (Group I or II) was determined based on proteolysis of cooked-meat media (CMM) pellets in pure culture (24). The California Department of Public Health (CDPH) identified the lone isolate of $C$. baratii type $\mathrm{F}$ based on this isolate's ability to produce lecithinase, as demonstrated on egg yolk agar; its inability to produce lipase; and its ability to produce type $\mathrm{F}$ toxin in broth culture, detected using the mouse bioassay. API20 A was used to confirm the identification of $C$. baratii type F. All animal use followed protocols approved by institutional (Health Canada or CDPH) animal care and use committees.

\section{Morbidity data}

Records on patient clinical information were retrieved from the 2005-2018 Discharge Abstract Database (DAD) and the 2005-2010 Hospital Morbidity Database (HMDB) of the Canadian Institute for Health Information (CIHI) by querying all records currently available that listed botulism in the first 10 suspected diagnostic codes (25). These records were then matched to records by age, sex, date of admission, date of sample and province/territory of residence. Only laboratory-confirmed cases were included in the analyses of the DAD and HMDB data.

\section{Ethics approval}

Formal ethics approval was not required because this study used de-identified healthcare data that were obtained under an agreement with $\mathrm{ClHI}$, and we report the results in aggregate form. 


\section{Results}

While botulism has been nationally notifiable in Canada since 1933, the first case of infant botulism was reported in Canada in 1979 (16), three years after the first cases were described in the US (4,5). From 1979 to 2019, there were 63 laboratory-confirmed cases of infant botulism in Canada (Figure 1), that is, an average of 1.6 cases per year. The Health Canada BRS confirmed 44 cases, while the BCCDC confirmed 19 cases. These cases of infant botulism are further described as one complete dataset and will be referred to as "laboratory-confirmed".

Figure 1: Number of laboratory-confirmed infant botulism cases in Canada, 1979-2019

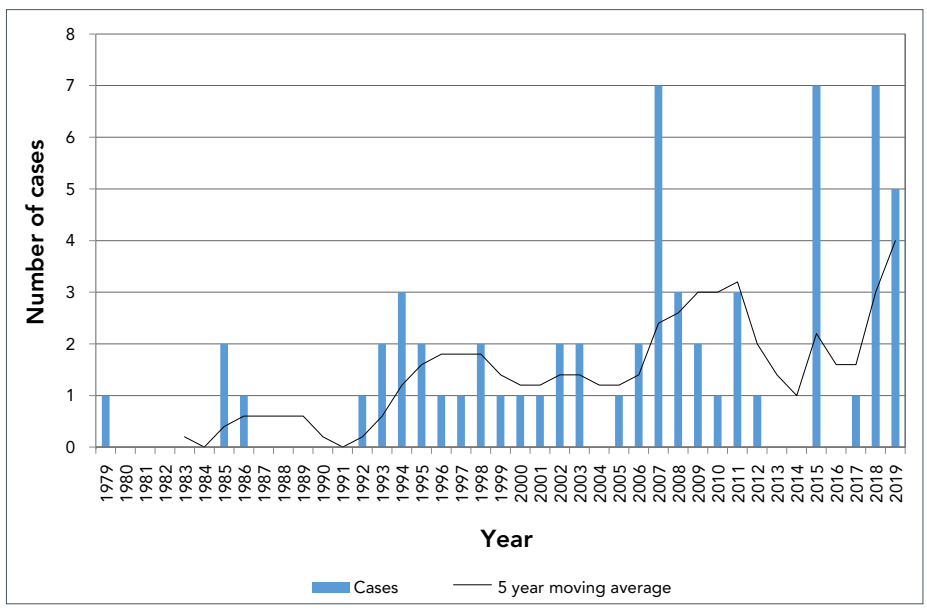

Infant botulism is a rare disease; the average annual incidence was calculated at 4.3 cases per million live births in Canada during this time period (Table 1). Confirmed cases occurred in Ontario $(n=21)$, British Columbia $(n=19)$, Québec $(n=12)$, Alberta $(n=8)$, Newfoundland and Labrador $(n=2)$ and Nova Scotia $(n=1)$ (Table 1). Of the 58 records that indicated sex, 34 were female (59\%) and 24 were male (41\%).

Table 1: Laboratory-confirmed infant botulism cases by serotype and province/territory, 1979-2019

\begin{tabular}{|c|c|c|c|c|c|c|}
\hline \multirow{2}{*}{$\begin{array}{l}\text { Province/ } \\
\text { territory }\end{array}$} & \multicolumn{4}{|c|}{$\begin{array}{c}\text { Cases by BoNT } \\
\text { serotype }\end{array}$} & \multirow[t]{2}{*}{ Total } & \multirow{2}{*}{$\begin{array}{c}\text { Annual rate/ } \\
\text { million live } \\
\text { births }^{\mathrm{a}}\end{array}$} \\
\hline & A & B & AB & $F$ & & \\
\hline Ontario & 11 & 9 & 1 & 0 & 21 & 3.75 \\
\hline $\begin{array}{l}\text { British } \\
\text { Columbia }\end{array}$ & 19 & 0 & 0 & 0 & 19 & 10.86 \\
\hline Québec & 9 & 2 & 0 & 1 & 12 & 3.63 \\
\hline Alberta & 8 & 0 & 0 & 0 & 8 & 4.41 \\
\hline $\begin{array}{l}\text { Newfoundland } \\
\text { and Labrador }\end{array}$ & 1 & 1 & 0 & 0 & 2 & 9.93 \\
\hline Nova Scotia & 0 & 1 & 0 & 0 & 1 & 2.66 \\
\hline Canada $^{b}$ & 48 & 13 & 1 & 1 & 63 & 4.30 \\
\hline
\end{tabular}

Abbreviation: BoNT, botulinum neurotoxin

Annual rate per million live births was calculated as the total case count divided by the average number of annual live births from 1991-2018 multiplied by 40 (years), divided by a million $(21,22)$ b No laboratory-confirmed cases of infant botulism in Manitoba, New Brunswick,

Northwest Territories, Nunavut, Prince Edward Island, Saskatchewan or Yukon
In comparison to the laboratory-confirmed cases, the CNDSS reported 57 cases of infant botulism from 1983 to 2018, giving an average of 1.6 cases per year. Considering the single laboratory-confirmed case in 1979 and the five laboratory-confirmed cases in 2019 , the total number of cases reported to the CNDSS matches the total number of 57 laboratory-confirmed cases from 1983 to 2018. However, the number of cases reported by the provinces and territories differed. Cases were reported to the CNDSS from Ontario $(n=22)$, British Columbia $(n=14)$, Québec $(n=10)$, Alberta $(n=9)$, Newfoundland and Labrador $(n=1)$ and Nova Scotia $(n=1)$. Of the 57 cases, 36 were female and 21 male. From 1983 to 2018, the laboratory-confirmed cases were from Ontario $(n=19)$, British Columbia ( $n=17)$, Québec $(n=11)$, Alberta $(n=8)$, Newfoundland and Labrador $(n=2)$, and Nova Scotia $(n=1)$.

The age of onset follows a unimodal distribution with mean 17.8 weeks and median 16 weeks (Figure 2). The youngest infant was two weeks of age, and the oldest infant was 52 weeks old. BoNT type A constituted the majority of cases ( $n=48 ; 76 \%)$, followed by type $B(n=13 ; 21 \%)$, type $A B(n=1 ; 2 \%)$ and type $F$ $(n=1 ; 2 \%)$ (Table 1). The single type $F$ case was identified as caused by $C$. baratii; this was the youngest infant, with an age of onset of two weeks. All type A strains of $C$. botulinum are Group I (proteolytic), while type B strains may be either Group I or II (non-proteolytic). Of the type B strains isolated and tested for proteolytic activity, all $(n=10)$ were classified as Group I C. botulinum.

\section{Figure 2: Age of onset for laboratory-confirmed infant botulism cases, Canada, 1979-2019}

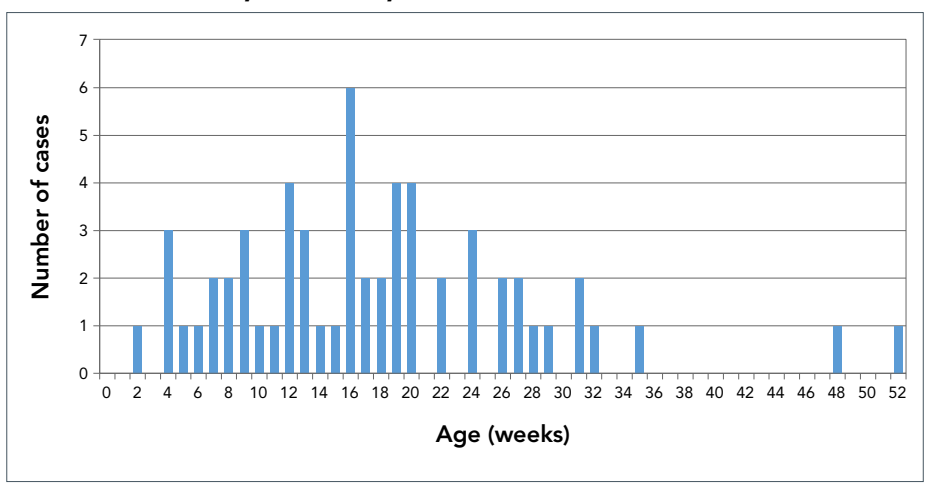

Of the 63 cases identified from 1979 to 2019,29 (46\%) had environmental or food samples submitted for testing and only six were matched with environmental samples, including honey $(n=4)$ and crib dust $(n=1)$, as well as a change mat swab and a washing tub swab $(n=1)$ (Table 2). Of the 58 records that were available, stool was by far the best clinical specimen for detection of BoNT $(n=55 ; 95 \%)$ and viable C. botulinum $(n=58$; $100 \%$ ) (Table 3). BoNT was detected in only 3 of 33 (9\%) serum specimens tested, while neither BoNT nor C. botulinum were detected in three specimens of gastric contents tested. 
Table 2: Laboratory-confirmed source attribution of infant botulism

\begin{tabular}{|c|l|l|}
\hline Year & Province/territory & \multicolumn{1}{c|}{ Source } \\
\hline 1985 & Québec & Honey \\
\hline 1993 & Alberta & Honey \\
\hline 1995 & Québec & Honey \\
\hline 2000 & British Columbia & Honey \\
\hline 2005 & Ontario & Crib dust \\
\hline 2009 & Québec & $\begin{array}{l}\text { Change mat swab and washing tub } \\
\text { swab }\end{array}$ \\
\hline
\end{tabular}

Table 3: Laboratory detection of BoNT and viable Clostridium botulinum in clinical specimens

\begin{tabular}{|l|r|r|r|r|r|}
\multirow{2}{*}{ Specimen } & \multirow{2}{*}{ Tested $(\mathbf{n})$} & \multicolumn{2}{|c|}{$\begin{array}{c}\text { BoNT } \\
\text { detected }\end{array}$} & \multicolumn{2}{|c|}{$\begin{array}{c}\text { C. botulinum } \\
\text { detected }\end{array}$} \\
\cline { 3 - 7 } & & $\mathbf{n}$ & \multicolumn{1}{c|}{ \% } & \multicolumn{1}{|c|}{$\mathbf{n}$} & \multicolumn{2}{c|}{$\%$} \\
\hline $\begin{array}{l}\text { Gastric } \\
\text { contents }\end{array}$ & 3 & 0 & 0 & 0 & 0 \\
\hline Serum & 33 & 3 & 9 & 0 & 0 \\
\hline Stool & 58 & 55 & 95 & 58 & 100 \\
\hline
\end{tabular}

Abbreviation: BoNT, botulinum neurotoxin

Of the 63 laboratory-confirmed infant botulism cases, 23 were cross-referenced to the 2005-2018 DAD and 2005-2010 HMDB. The mean (standard deviation [SD]) length of hospital stay of these patients was 20.3 (7.0) days (Table 4). Over a half $(n=13)$ were transferred to special care units. These special care units are specifically designed, staffed and equipped for the continuous observation and treatment of patients who cannot be cared for in a general acute care unit. These include intensive care units and step-down units (25). The patients spent a mean (SD) of 12.2 (6.3) days in a special care unit; eight needed a ventilator (Table 4). Most were discharged to the patient's home with either no support $(n=9)$ or with the support of home care workers $(n=10)$. Three were transferred to acute care during hospitalization; the discharge disposition of one patient was unknown (Table 5). No deaths were reported for the 23 cross-referenced cases.

Table 4: Hospital metrics for laboratory-confirmed hospitalization cases $(n=23)$

\begin{tabular}{|l|r|r|r|}
\multicolumn{1}{|c|}{$\begin{array}{c}\text { Hospital } \\
\text { metric }\end{array}$} & Mean & \multicolumn{1}{|c|}{$\begin{array}{c}\text { Standard } \\
\text { deviation }\end{array}$} & \multicolumn{2}{c|}{$\begin{array}{c}\text { Number of } \\
\text { cases }\end{array}$} \\
\hline $\begin{array}{l}\text { Length of stay } \\
\text { (days) }\end{array}$ & 20.3 & 7.0 & 23 \\
\hline $\begin{array}{l}\text { Time in special } \\
\text { care (days) }\end{array}$ & 12.2 & 6.3 & 13 \\
\hline $\begin{array}{l}\text { Use of } \\
\text { ventilator }\end{array}$ & N/A & N/A & 8 \\
\hline
\end{tabular}

Table 5: Clinical outcome of laboratory-confirmed hospitalization cases $(n=23)$

\begin{tabular}{|l|r|}
\multicolumn{1}{|c|}{ Discharge disposition } & \multicolumn{2}{c|}{ Number of cases } \\
\hline Home, no support & 9 \\
\hline Home with support & 10 \\
\hline Transferred to acute care & 3 \\
\hline Unknown & 1 \\
\hline Death & 0 \\
\hline
\end{tabular}

\section{Discussion}

From 1979 to 2019 there were 63 laboratory-confirmed cases of infant botulism in Canada. From 1983 to 2018, 57 cases were reported to the CNDSS, which matched the total number of laboratory-confirmed cases during this time period, however the provinces and territories reporting the cases differed. The reasons for the discrepancies is unknown; however, this discrepancy is not unexpected, as the reference laboratories are directly involved in case diagnosis while public health authorities may not be involved in every sporadic case. In recent years, the BRS for Canada and the Public Health Agency of Canada have collaborated to ensure more complete reporting to the appropriate public health authorities.

The annual incidence rate of 4.3 cases per million live births in Canada is similar to incidence rates reported in Australia (4.4 cases per million live births), Italy (2.1 cases per million live births) and Denmark (6.7 cases per million live births) and less than the rates reported in the US (20.3 cases per million live births) and Argentina (24.1 cases per million live births) (26).

The average age at onset was 17.8 weeks in Canada. This is higher than the reported mean ages of onset of 13.8 weeks in the US and 14.3 weeks globally outside the US (26). The age of onset may correspond to changes in gut microbiota after weaning (27) that leaves the gut more susceptible to colonization. A recent study found that breast-fed infant botulism patients were older at onset than formula-fed patients (28), although the role of breast feeding in infant botulism is controversial $(29,30)$.

The finding that $98 \%$ of the cases were types $A, B$ or $A B$ is consistent with a report that $C$. botulinum types $A$ and $B$ accounted for $98.7 \%$ of all recorded infant botulism cases worldwide from 1978 to 2006 (26). The single case of type $\mathrm{F}$ identified as being caused by $C$. baratii is rare but not unprecedented. Cases of infant botulism caused by toxigenic strains of $C$. butyricum type $E(8-10)$ and $C$. baratii type $F(11-14)$, as well as a single case caused by $C$. botulinum type $E(31)$, have been described elsewhere. The predominance of $C$. botulinum type $A$ in the western US correlates with the occurrence of only type A cases in the two western provinces, Alberta and British Columbia (Table 1) (32). 
Likewise, 28 instances from the $\mathrm{CIHI}$ database were coded as "botulism" based on differential diagnosis, yet samples were not sent to a laboratory for confirmation. Finally, there were several instances of laboratory-confirmed infant botulism cases that could not be identified in the $\mathrm{ClHI}$ databases, even within the reported years. Considering a recent summary of 1,345 infant botulism cases in California from 1976-2016 found that $99.3 \%$ of cases were hospitalized (36), it is likely that the missing hospital records for laboratory-confirmed cases in Canada were the result of a missing diagnostic code in the $\mathrm{CIHI}$ database. Improved communication between hospitals, public health officials and diagnostic laboratories would help to capture all cases of infant botulism in Canada and help in the diagnosis and evaluation of treatments. Physicians and hospital staff can refer to the Canadian botulism guide for healthcare professionals for instructions on differential diagnosis, proper sampling of clinical specimens and treatment with BabyBIG antitoxin (37).

\section{Conclusion}

Infant botulism is a rare disease in Canada. The unimodal distribution for age of onset suggests a temporal susceptibility to colonization by $C$. botulinum which peaks at 16 weeks. Although no deaths were reported in this time period, the average length of stay in the hospital and the number of cases transferred to special care demonstrate severe clinical outcomes for patients suffering from this disease. The effectiveness of the BabyBIG antitoxin could not be evaluated due to limitations in reporting and should be addressed in the future.

\section{Authors' statement}

$\mathrm{RH}$ - Examined the laboratory records of the Health Canada Botulism Reference Service; queried records of the Discharge Abstract Database and the Hospital Morbidity Database of the Canadian Institute of Health Information for data on hospitalization of infant botulism cases; performed all statistical analyses; performed additional literature searches; wrote the first draft

CT - Examined the laboratory records of the British Columbia Public Health Reference Microbiology Laboratory; commented on-approved the manuscript

NP - Examined the laboratory records of the British Columbia Public Health Reference Microbiology Laboratory; commented on-approved the manuscript

JC - Queried the Canadian Notifiable Disease Surveillance System for data on reported cases of infant botulism, wrote the section on the Canadian Disease Surveillance System and commented on-approved the manuscript

JA - Conceived the summary, examined laboratory records of the Health Canada Botulism Reference Service, performed additional literature searches, drafted certain sections and revised the article

\section{Competing interests}

None.
The sporadic nature of infant botulism and the ubiquity of
For laboratory detection of BoNT or isolation of viable
C. botulinum, the proper collection and handling of clinical
specimens is essential. Of the 58 records that were available,
stool was by far the best clinical specimen for detection of
BoNT ( $95 \%$ ) and viable C. botulinum (100\%). This is consistent
with a previous report that stool or enema effluent are preferred
diagnostic specimens for infant botulism (34).
Of the 23 infant botulism cases that were cross-referenced to the
DAD and HMDB databases, 13 ( $57 \%$ ) were transferred to special
care and eight ( $35 \%$ ) needed ventilator support. No deaths were
reported. This is consistent with previous data indicating that
infant botulism is a severe disease requiring intervention in the
majority of cases. Outside the US, the percentage of reported
cases who required ventilator or intubation support was $67 \%$ and
deaths accounted for $1.1 \%$ of cases (26). In the US, $56 \%$ of
hospitalized infant botulism cases required ventilator support or
intubation during a trial of BabyBIG (15), while the mortality rate
in the US is less than $1 \%$ ( 35 ).
Limitations
The narrow range of hospital records available from CIHI
databases ( $2005-2018$ for DAD and $2005-2010$ for HMDB)
reduced the number of cases that could be linked to the
laboratory records and lowered the statistical power of data
obtained from the hospital records, such as severity of illness and
is not routinely recorded in the CIHI database; we recommend
that this be included to properly evaluate the effect of this
thent.

For laboratory detection of BoNT or isolation of viable
C. botulinum, the proper collection and handling of clinical
specimens is essential. Of the 58 records that were available,
stool was by far the best clinical specimen for detection of
BoNT ( $95 \%$ ) and viable C. botulinum (100\%). This is consistent
with a previous report that stool or enema effluent are preferred
diagnostic specimens for infant botulism (34).
Of the 23 infant botulism cases that were cross-referenced to the
DAD and HMDB databases, 13 ( $57 \%$ ) were transferred to special
care and eight ( $35 \%$ ) needed ventilator support. No deaths were
reported. This is consistent with previous data indicating that
infant botulism is a severe disease requiring intervention in the
majority of cases. Outside the US, the percentage of reported
cases who required ventilator or intubation support was $67 \%$ and
deaths accounted for $1.1 \%$ of cases (26). In the US, $56 \%$ of
hospitalized infant botulism cases required ventilator support or
intubation during a trial of BabyBIG (15), while the mortality rate
in the US is less than $1 \%$ ( 35 ).
Limitations
The narrow range of hospital records available from CIHI
databases ( $2005-2018$ for DAD and $2005-2010$ for HMDB)
reduced the number of cases that could be linked to the
laboratory records and lowered the statistical power of data
obtained from the hospital records, such as severity of illness and
is not routinely recorded in the CIHI database; we recommend
that this be included to properly evaluate the effect of this
thent.

For laboratory detection of BoNT or isolation of viable
C. botulinum, the proper collection and handling of clinical
specimens is essential. Of the 58 records that were available,
stool was by far the best clinical specimen for detection of
BoNT ( $95 \%$ ) and viable C. botulinum (100\%). This is consistent
with a previous report that stool or enema effluent are preferred
diagnostic specimens for infant botulism (34).
Of the 23 infant botulism cases that were cross-referenced to the
DAD and HMDB databases, 13 ( $57 \%$ ) were transferred to special
care and eight ( $35 \%$ ) needed ventilator support. No deaths were
reported. This is consistent with previous data indicating that
infant botulism is a severe disease requiring intervention in the
majority of cases. Outside the US, the percentage of reported
cases who required ventilator or intubation support was $67 \%$ and
deaths accounted for $1.1 \%$ of cases (26). In the US, $56 \%$ of
hospitalized infant botulism cases required ventilator support or
intubation during a trial of BabyBIG (15), while the mortality rate
in the US is less than $1 \%$ ( 35 ).
Limitations
The narrow range of hospital records available from CIHI
databases ( $2005-2018$ for DAD and $2005-2010$ for HMDB)
reduced the number of cases that could be linked to the
laboratory records and lowered the statistical power of data
obtained from the hospital records, such as severity of illness and
is not routinely recorded in the CIHI database; we recommend
that this be included to properly evaluate the effect of this
thent.

For laboratory detection of BoNT or isolation of viable
C. botulinum, the proper collection and handling of clinical
specimens is essential. Of the 58 records that were available,
stool was by far the best clinical specimen for detection of
BoNT ( $95 \%$ ) and viable C. botulinum (100\%). This is consistent
with a previous report that stool or enema effluent are preferred
diagnostic specimens for infant botulism (34).
Of the 23 infant botulism cases that were cross-referenced to the
DAD and HMDB databases, 13 ( $57 \%$ ) were transferred to special
care and eight ( $35 \%$ ) needed ventilator support. No deaths were
reported. This is consistent with previous data indicating that
infant botulism is a severe disease requiring intervention in the
majority of cases. Outside the US, the percentage of reported
cases who required ventilator or intubation support was $67 \%$ and
deaths accounted for $1.1 \%$ of cases (26). In the US, $56 \%$ of
hospitalized infant botulism cases required ventilator support or
intubation during a trial of BabyBIG (15), while the mortality rate
in the US is less than $1 \%$ ( 35 ).
Limitations
The narrow range of hospital records available from CIHI
databases ( $2005-2018$ for DAD and $2005-2010$ for HMDB)
reduced the number of cases that could be linked to the
laboratory records and lowered the statistical power of data
obtained from the hospital records, such as severity of illness and
is not routinely recorded in the CIHI database; we recommend
that this be included to properly evaluate the effect of this
thent.

For laboratory detection of BoNT or isolation of viable
C. botulinum, the proper collection and handling of clinical
specimens is essential. Of the 58 records that were available,
stool was by far the best clinical specimen for detection of
BoNT ( $95 \%$ ) and viable C. botulinum (100\%). This is consistent
with a previous report that stool or enema effluent are preferred
diagnostic specimens for infant botulism (34).
Of the 23 infant botulism cases that were cross-referenced to the
DAD and HMDB databases, 13 ( $57 \%$ ) were transferred to special
care and eight ( $35 \%$ ) needed ventilator support. No deaths were
reported. This is consistent with previous data indicating that
infant botulism is a severe disease requiring intervention in the
majority of cases. Outside the US, the percentage of reported
cases who required ventilator or intubation support was $67 \%$ and
deaths accounted for $1.1 \%$ of cases (26). In the US, $56 \%$ of
hospitalized infant botulism cases required ventilator support or
intubation during a trial of BabyBIG (15), while the mortality rate
in the US is less than $1 \%$ ( 35 ).
Limitations
The narrow range of hospital records available from CIHI
databases ( $2005-2018$ for DAD and $2005-2010$ for HMDB)
reduced the number of cases that could be linked to the
laboratory records and lowered the statistical power of data
obtained from the hospital records, such as severity of illness and
is not routinely recorded in the CIHI database; we recommend
that this be included to properly evaluate the effect of this
thent.

For laboratory detection of BoNT or isolation of viable
C. botulinum, the proper collection and handling of clinical
specimens is essential. Of the 58 records that were available,
stool was by far the best clinical specimen for detection of
BoNT ( $95 \%$ ) and viable C. botulinum (100\%). This is consistent
with a previous report that stool or enema effluent are preferred
diagnostic specimens for infant botulism (34).
Of the 23 infant botulism cases that were cross-referenced to the
DAD and HMDB databases, 13 ( $57 \%$ ) were transferred to special
care and eight ( $35 \%$ ) needed ventilator support. No deaths were
reported. This is consistent with previous data indicating that
infant botulism is a severe disease requiring intervention in the
majority of cases. Outside the US, the percentage of reported
cases who required ventilator or intubation support was $67 \%$ and
deaths accounted for $1.1 \%$ of cases (26). In the US, $56 \%$ of
hospitalized infant botulism cases required ventilator support or
intubation during a trial of BabyBIG (15), while the mortality rate
in the US is less than $1 \%$ ( 35 ).
Limitations
The narrow range of hospital records available from CIHI
databases ( $2005-2018$ for DAD and $2005-2010$ for HMDB)
reduced the number of cases that could be linked to the
laboratory records and lowered the statistical power of data
obtained from the hospital records, such as severity of illness and
is not routinely recorded in the CIHI database; we recommend
that this be included to properly evaluate the effect of this
thent.

For laboratory detection of BoNT or isolation of viable
C. botulinum, the proper collection and handling of clinical
specimens is essential. Of the 58 records that were available,
stool was by far the best clinical specimen for detection of
BoNT ( $95 \%$ ) and viable C. botulinum (100\%). This is consistent
with a previous report that stool or enema effluent are preferred
diagnostic specimens for infant botulism (34).
Of the 23 infant botulism cases that were cross-referenced to the
DAD and HMDB databases, 13 ( $57 \%$ ) were transferred to special
care and eight ( $35 \%$ ) needed ventilator support. No deaths were
reported. This is consistent with previous data indicating that
infant botulism is a severe disease requiring intervention in the
majority of cases. Outside the US, the percentage of reported
cases who required ventilator or intubation support was $67 \%$ and
deaths accounted for $1.1 \%$ of cases (26). In the US, $56 \%$ of
hospitalized infant botulism cases required ventilator support or
intubation during a trial of BabyBIG (15), while the mortality rate
in the US is less than $1 \%$ ( 35 ).
Limitations
The narrow range of hospital records available from CIHI
databases ( $2005-2018$ for DAD and $2005-2010$ for HMDB)
reduced the number of cases that could be linked to the
laboratory records and lowered the statistical power of data
obtained from the hospital records, such as severity of illness and
is not routinely recorded in the CIHI database; we recommend
that this be included to properly evaluate the effect of this
thent.

For laboratory detection of BoNT or isolation of viable
C. botulinum, the proper collection and handling of clinical
specimens is essential. Of the 58 records that were available,
stool was by far the best clinical specimen for detection of
BoNT ( $95 \%$ ) and viable C. botulinum (100\%). This is consistent
with a previous report that stool or enema effluent are preferred
diagnostic specimens for infant botulism (34).
Of the 23 infant botulism cases that were cross-referenced to the
DAD and HMDB databases, 13 (57\%) were transferred to special
care and eight ( $35 \%$ ) needed ventilator support. No deaths were
reported. This is consistent with previous data indicating that
infant botulism is a severe disease requiring intervention in the
majority of cases. Outside the US, the percentage of reported
cases who required ventilator or intubation support was $67 \%$ and
deaths accounted for $1.1 \%$ of cases (26). In the US, $56 \%$ of
hospitalized infant botulism cases required ventilator support or
intubation during a trial of BabyBIG (15), while the mortality rate
in the US is less than $1 \%$ ( 35 ).
Limitations
The narrow range of hospital records available from CIHI
databases (2005-2018 for DAD and 2005-2010 for HMDB)
reduced the number of cases that could be linked to the
laboratory records and lowered the statistical power of data
obtained from the hospital records, such as severity of illness and
efficacy of treatment. Of note, treatment with BabyBIG antitoxin
is not routinely recorded in the CIHI database; we recommend
that this be included to properly evaluate the effect of this
treatment.

For laboratory detection of BoNT or isolation of viable
C. botulinum, the proper collection and handling of clinical
specimens is essential. Of the 58 records that were available,
stool was by far the best clinical specimen for detection of
BoNT ( $95 \%$ ) and viable C. botulinum (100\%). This is consistent
with a previous report that stool or enema effluent are preferred
diagnostic specimens for infant botulism (34).
Of the 23 infant botulism cases that were cross-referenced to the
DAD and HMDB databases, 13 ( $57 \%$ ) were transferred to special
care and eight ( $35 \%$ ) needed ventilator support. No deaths were
reported. This is consistent with previous data indicating that
infant botulism is a severe disease requiring intervention in the
majority of cases. Outside the US, the percentage of reported
cases who required ventilator or intubation support was $67 \%$ and
deaths accounted for $1.1 \%$ of cases (26). In the US, $56 \%$ of
hospitalized infant botulism cases required ventilator support or
intubation during a trial of BabyBIG (15), while the mortality rate
in the US is less than $1 \%$ ( 35 ).
Limitations
The narrow range of hospital records available from CIHI
databases ( $2005-2018$ for DAD and $2005-2010$ for HMDB)
reduced the number of cases that could be linked to the
laboratory records and lowered the statistical power of data
obtained from the hospital records, such as severity of illness and
is not routinely recorded in the CIHI database; we recommend
that this be included to properly evaluate the effect of this
thent.

For laboratory detection of BoNT or isolation of viable
C. botulinum, the proper collection and handling of clinical
specimens is essential. Of the 58 records that were available,
stool was by far the best clinical specimen for detection of
BoNT ( $95 \%$ ) and viable C. botulinum (100\%). This is consistent
with a previous report that stool or enema effluent are preferred
diagnostic specimens for infant botulism (34).
Of the 23 infant botulism cases that were cross-referenced to the
DAD and HMDB databases, 13 ( $57 \%$ ) were transferred to special
care and eight ( $35 \%$ ) needed ventilator support. No deaths were
reported. This is consistent with previous data indicating that
infant botulism is a severe disease requiring intervention in the
majority of cases. Outside the US, the percentage of reported
cases who required ventilator or intubation support was $67 \%$ and
deaths accounted for $1.1 \%$ of cases (26). In the US, $56 \%$ of
hospitalized infant botulism cases required ventilator support or
intubation during a trial of BabyBIG (15), while the mortality rate
in the US is less than $1 \%$ ( 35 ).
Limitations
The narrow range of hospital records available from CIHI
databases ( $2005-2018$ for DAD and $2005-2010$ for HMDB)
reduced the number of cases that could be linked to the
laboratory records and lowered the statistical power of data
obtained from the hospital records, such as severity of illness and
is not routinely recorded in the CIHI database; we recommend
that this be included to properly evaluate the effect of this
thent.

For laboratory detection of BoNT or isolation of viable
C. botulinum, the proper collection and handling of clinical
specimens is essential. Of the 58 records that were available,
stool was by far the best clinical specimen for detection of
BoNT ( $95 \%$ ) and viable C. botulinum (100\%). This is consistent
with a previous report that stool or enema effluent are preferred
diagnostic specimens for infant botulism (34).
Of the 23 infant botulism cases that were cross-referenced to the
DAD and HMDB databases, 13 ( $57 \%$ ) were transferred to special
care and eight ( $35 \%$ ) needed ventilator support. No deaths were
reported. This is consistent with previous data indicating that
infant botulism is a severe disease requiring intervention in the
majority of cases. Outside the US, the percentage of reported
cases who required ventilator or intubation support was $67 \%$ and
deaths accounted for $1.1 \%$ of cases (26). In the US, $56 \%$ of
hospitalized infant botulism cases required ventilator support or
intubation during a trial of BabyBIG (15), while the mortality rate
in the US is less than $1 \%$ ( 35 ).
Limitations
The narrow range of hospital records available from CIHI
databases ( $2005-2018$ for DAD and $2005-2010$ for HMDB)
reduced the number of cases that could be linked to the
laboratory records and lowered the statistical power of data
obtained from the hospital records, such as severity of illness and
is not routinely recorded in the CIHI database; we recommend
that this be included to properly evaluate the effect of this
thent.

For laboratory detection of BoNT or isolation of viable
C. botulinum, the proper collection and handling of clinical
specimens is essential. Of the 58 records that were available,
stool was by far the best clinical specimen for detection of
BoNT ( $95 \%$ ) and viable C. botulinum (100\%). This is consistent
with a previous report that stool or enema effluent are preferred
diagnostic specimens for infant botulism (34).
Of the 23 infant botulism cases that were cross-referenced to the
DAD and HMDB databases, 13 ( $57 \%$ ) were transferred to special
care and eight ( $35 \%$ ) needed ventilator support. No deaths were
reported. This is consistent with previous data indicating that
infant botulism is a severe disease requiring intervention in the
majority of cases. Outside the US, the percentage of reported
cases who required ventilator or intubation support was $67 \%$ and
deaths accounted for $1.1 \%$ of cases (26). In the US, $56 \%$ of
hospitalized infant botulism cases required ventilator support or
intubation during a trial of BabyBIG (15), while the mortality rate
in the US is less than $1 \%$ ( 35 ).
Limitations
The narrow range of hospital records available from CIHI
databases ( $2005-2018$ for DAD and $2005-2010$ for HMDB)
reduced the number of cases that could be linked to the
laboratory records and lowered the statistical power of data
obtained from the hospital records, such as severity of illness and
is not routinely recorded in the CIHI database; we recommend
that this be included to properly evaluate the effect of this
thent.

For laboratory detection of BoNT or isolation of viable
C. botulinum, the proper collection and handling of clinical
specimens is essential. Of the 58 records that were available,
stool was by far the best clinical specimen for detection of
BoNT ( $95 \%$ ) and viable C. botulinum (100\%). This is consistent
with a previous report that stool or enema effluent are preferred
diagnostic specimens for infant botulism (34).
Of the 23 infant botulism cases that were cross-referenced to the
DAD and HMDB databases, 13 ( $57 \%$ ) were transferred to special
care and eight ( $35 \%$ ) needed ventilator support. No deaths were
reported. This is consistent with previous data indicating that
infant botulism is a severe disease requiring intervention in the
majority of cases. Outside the US, the percentage of reported
cases who required ventilator or intubation support was $67 \%$ and
deaths accounted for $1.1 \%$ of cases (26). In the US, $56 \%$ of
hospitalized infant botulism cases required ventilator support or
intubation during a trial of BabyBIG (15), while the mortality rate
in the US is less than $1 \%$ ( 35 ).
Limitations
The narrow range of hospital records available from CIHI
databases ( $2005-2018$ for DAD and $2005-2010$ for HMDB)
reduced the number of cases that could be linked to the
laboratory records and lowered the statistical power of data
obtained from the hospital records, such as severity of illness and
is not routinely recorded in the CIHI database; we recommend
that this be included to properly evaluate the effect of this
thent.$$
\text { treatment. }
$$ 


\section{Acknowledgements}

The authors wish to acknowledge J Barash and S Arnon of the California Department of Public Health Infant Botulism Treatment and Prevention Program (CDPH/IBTPP) for isolation and identification of $C$. baratii type $F$ as the causative agent of the single case of type $F$ infant botulism from a stool specimen sent to $\mathrm{CDPH} / \mathrm{IBTPP}$ for research purposes.

\section{Funding}

This work was supported by Health Canada, the British Columbia Centre for Disease Control (BCCDC) Public Health Laboratory, and the Public Health Agency of Canada.

\section{References}

1. Fleck-Derderian $S$, Shankar $M$, Rao AK, Chatham-Stephens K, Adjei S, Sobel J, Meltzer MI, Meaney-Delman D, Pillai SK. The epidemiology of foodborne botulism outbreaks: a systematic review. Clin Infect Dis 2017;66(suppl_1):S73-81. DOI

2. Chatham-Stephens K, Fleck-Derderian S, Johnson SD, Sobel J, Rao AK, Meaney-Delman D. Clinical features of foodborne and wound botulism: a systematic review of the literature, 1932-2015. Clin Infect Dis 2017;66(suppl_1):S11-6. DOI

3. Harris RA, Anniballi F, Austin JW. Adult intestinal toxemia botulism. Toxins (Basel) 2020;12(2):81. DOI PubMed

4. Pickett J, Berg B, Chaplin E, Brunstetter-Shafer MA. Syndrome of botulism in infancy: clinical and electrophysiologic study. N Engl J Med 1976;295(14):770-2. DOI PubMed

5. Midura TF, Arnon SS. Infant botulism. Identification of Clostridium botulinum and its toxins in faeces. Lancet 1976;308(7992):934-6. DOI PubMed

6. Arnon SS, Damus K, Chin J. Infant botulism: epidemiology and relation to sudden infant death syndrome. Epidemiol Rev 1981;3:45-66. DOI PubMed

7. Arnon SS. Infant botulism. Annu Rev Med 1980;31:541-60. DOI PubMed

8. Fenicia L, Da Dalt L, Anniballi F, Franciosa G, Zanconato S, Aureli P. A case if infant botulism due to neurotoxigenic Clostridium butyricum type E associated with Clostridium difficile colitis. Eur J Clin Microbiol Infect Dis 2002;21(10):736-8. DOI PubMed

9. McCroskey LM, Hatheway CL, Fenicia L, Pasolini B, Aureli P. Characterization of an organism that produces type $\mathrm{E}$ botulinal toxin but which resembles Clostridium butyricum from the feces of an infant with type $E$ botulism. J Clin Microbiol 1986;23(1):201-2. DOI PubMed
10. Aureli $P$, Fenicia $L$, Pasolini B, Gianfranceschi $M$, McCroskey LM, Hatheway CL. Two cases of type E infant botulism caused by neurotoxigenic Clostridium butyricum in Italy. J Infect Dis 1986;154(2):207-11. DOI PubMed

11. Paisley JW, Lauer BA, Arnon SS. A second case of infant botulism type $\mathrm{F}$ caused by Clostridium baratii. Pediatr Infect Dis J 1995;14(10):912-4. DOI PubMed

12. Barash JR, Tang TW, Arnon SS. First case of infant botulism caused by Clostridium baratii type $\mathrm{F}$ in California. J Clin Microbiol 2005;43(8):4280-2. DOI PubMed

13. Halpin AL, Khouri JM, Payne JR, Nakao JH, Cronquist A, Kalas N, Mohr M, Osborne M, O'Dell S, Luquez C, Klontz KC, Sobel J, Rao AK. Type F infant botulism: Investigation of recent clusters and overview of this exceedingly rare disease. Clin Infect Dis 2017;66(suppl_1):S92-4. DOI

14. Moodley A, Quinlisk P, Garvey A, Kalas N, Barash JR, Khouri JM; Centers for Disease Control and Prevention (CDC). Notes from the field: infant botulism caused by Clostridium baratii type F - lowa, 2013. MMWR Morb Mortal Wkly Rep 2015;64(14):400. PubMed

15. Arnon SS, Schechter R, Maslanka SE, Jewell NP, Hatheway CL. Human botulism immune globulin for the treatment of infant botulism. N Engl J Med 2006;354(5):462-71. DOI PubMed

16. McCurdy DM, Krishnan $\mathrm{C}$, Hauschild $\mathrm{AH}$. Infant botulism in Canada. Can Med Assoc J 1981;125(7):741-3. PubMed

17. Roland EH, Ebelt VJ, Anderson JD, Hill A. Infant botulism: a rare entity in Canada? CMAJ 1986;135(2):130-1. PubMed

18. Siu K, Rehan M, Austin JW, Ramachandran Nair R, Pernica J. It's not all about the honey. Paediatr Child Health 2017;22(1):37-8. DOI PubMed

19. Hauschild AH, Bowmer EJ, Gauvreau L. Infant botulism. Can Med Assoc J 1978;118(5):484. PubMed

20. Schwartz KL, Austin JW, Science M. Constipation and poor feeding in an infant with botulism. CMAJ 2012;184(17):1919-22. DOI PubMed

21. Statistics Canada. Census profile 2016 [Internet]. Ottawa (ON): Statistics Canada; (updated 2021-03-11; accessed 2020-09-28). https://www12.statcan.gc.ca/censusrecensement/index-eng.cfm

22. United Nations Statistics Division. UN data [Internet]. New York (NY): UNSD; (accessed 2020-09-28). http://data.un.org/ en/iso/ca.htm

23. National case definition: botulism [Internet]. Ottawa (ON): Government of Canada; (updated 2019-11-26; accessed 2020-12-22). https://www.canada.ca/en/public-health/ services/diseases/botulism/professionals/national-casedefinition.htm 
24. Austin J, Sanders G. HPB methods for the microbiological analysis of foods, Volume 2: detection of Clostridium botulinum and its toxins in suspect foods and clinical specimens. 2009 (updated 2018-05-09; accessed 2020-1222). https://www.canada.ca/en/health-canada/services/foodnutrition/research-programs-analytical-methods/analyticalmethods/compendium-methods/methods-microbiologicalanalysis-foods-compendium-analytical-methods.html

25. Canadian Institute for Health Information. Inpatient hospitalizations and average length of stay trends in Canada, 2003-2004 and 2004-2005. Analysis in brief. Ottawa (ON): $\mathrm{CIHI} ; 2005$. https://secure.cihi.ca/free_products/hmdb_ analysis_in_brief_e.pdf

26. Koepke R, Sobel J, Arnon SS. Global occurrence of infant botulism, 1976-2006. Pediatrics 2008;122(1):e73-82. DOl PubMed

27. Leong C, Haszard JJ, Lawley B, Otal A, Taylor RW, Szymlek-Gay EA, Fleming EA, Daniels L, Fangupo LJ, Tannock GW, Heath AM. Mediation analysis as a means of identifying dietary components that differentially affect the fecal microbiota of infants weaned by modified baby-led and traditional approaches. Appl Environ Microbiol 2018;84(18):e00914-8. DOl PubMed

28. Panditrao MV, Dabritz HA, Kazerouni NN, Damus KH, Meissinger JK, Arnon SS. Seven-year case-control study in California of risk factors for infant botulism. J Pediatr 2020;227:258-267.e8. DOI PubMed

29. Arnon SS, Damus K, Thompson B, Midura TF, Chin J. Protective role of human milk against sudden death from infant botulism. J Pediatr 1982;100(4):568-73. DOI PubMed
30. Spika JS, Shaffer N, Hargrett-Bean N, Collin S, MacDonald KL, Blake PA. Risk factors for infant botulism in the United States. Am J Dis Child 1989;143(7):828-32. DOI PubMed

31. Lúquez C, Dykes JK, Yu PA, Raphael BH, Maslanka SE. First report worldwide of an infant botulism case due to Clostridium botulinum type E. J Clin Microbiol 2010;48(1):326-8. DOI PubMed

32. Smith LD. The occurrence of Clostridium botulinum and Clostridium tetani in the soil of the United States. Health Lab Sci 1978;15(2):74-80. PubMed

33. Nevas $M$, Lindström $M$, Virtanen $A$, Hielm $S$, Kuusi $M$ Arnon SS, Vuori E, Korkeala $\mathrm{H}$. Infant botulism acquired from household dust presenting as sudden infant death syndrome. J Clin Microbiol 2005;43(1):511-3. DOI PubMed

34. Midura TF. Update: infant botulism. Clin Microbiol Rev 1996;9(2):119-25. DOI PubMed

35. Jackson KA, Mahon BE, Copeland J, Fagan RP. Botulism mortality in the USA, 1975-2009. Botulinum J 2015;3(1):6-17. DOI PubMed

36. Panditrao MV, Dabritz HA, Kazerouni NN, Damus $\mathrm{KH}_{\text {, }}$ Meissinger JK, Arnon SS. Descriptive epidemiology of infant botulism in California: the first 40 years. J Pediatr 2020;227:247-257.e3. DOI PubMed

37. Health Canada. Botulism - Guide for healthcare professionals [Internet]. Ottawa (ON): Health Canada; 2020 (accessed 2020-12-22). https://www.canada.ca/ en/health-canada/services/food-nutrition/legislationguidelines/guidance-documents/botulism-guide-healthcareprofessionals-2012.htm 